\title{
Yellow Rust Extraction in Wheat Crop based on Color Segmentation Techniques
}

\author{
Amina Khatra \\ ${ }^{I}$ BGIET, Sangrur, Punjab, INDIA
}

\begin{abstract}
The presented work presents a color based segmentation techniques for extraction of yellow rust in whet crop images. Accurate segmentation of yellow rust in wheat crop images is very part of assessment of disease penetration into the wheat crop. And in turn to o take the necessary preventive action for minimizing the crop damage. The jpeg images acquired from CCD camera are read into the matlab tool and a color based segmentation algorithm is performed to segment the yellow rust. The segmentation of color is performed base on k-means algorithm.
\end{abstract}

Keywords: - Yellow Rust, K-Means, Segmentation, Clustering

\section{INTRODUCTION}

There occurs a need for a simple and cost-effective optical device for remote disease detection, to assist in monitoring diseases in plants. In its most basic sense, remote sensing is the practice of gathering information about something without touching it. Photographic images of diseased plants can be called remote sensing. Machine vision is an area of research that is merging with remote sensing and finding use in agriculture. In machine vision, a computer analyzes an image and measures parameters within it. Groundbased uses of these fields allow greater precision and accuracy for disease assessment and quantification.

The key idea behind the presented work is to develop a novel technique for detection of any decease/infection from the leaves in wheat plants that suffers at a higher rate from yellow rust. The main problem in wheat plants leaves image capturing arises on account of cover area. For, in the present work, it is suggested to install no. of close circuit CCD cameras to cover small areas at critical positions in the field like in the centre, corners and centres. After capturing the image, the leaves images are brought under image processing techniques to identify the growth and type of infections.

\section{RELATED WORKS}

Various image processing and pattern recognition techniques have been attempted by researchers for developing a ground-based sensor system to assist in monitoring diseases in plants under field conditions. Machine vision is an area of research that is merging with remote sensing and finding use in agriculture [9].

Ying \& others studied methods of image preprocessing for recognition of crop diseases in [5]. They made a comparative study of effect of the simple filter and median filter. They reported that leaves with spots must be pre-processed firstly in order to carry out any intelligent diagnosis of crop disease based on image processing and appropriate features can be extracted on the basis of this.

Naser etal [1] presented the design and development of an expert system with two different methods for diagnosing plant diseases. The first one uses the descriptive method and the other one uses the Graphical representation method. It was found that the graphical representation method required lesser descriptions from users. The proposed method was a fast and convenient method for identifying plant diseases.

Hiary etal [6] in their paper have presented the applications of K-means clustering and Neural Networks (NNs) clustering and classification. Disease recognition was the main objective of the proposed approach. The proposed algorithm was tested on five diseases of cotton leaves.

Bhegin et.al. of Digital Imaging Research Centre, Kingston University, London, UK, [4] suggested to classify different species of leaves based on the shape and texture of the plant leaf. The shape-based method obtained the contour signature from every leaf and then calculated the variation between them using a divergence measure. The orientations of the edge gradients were used to evaluate the macro-texture of the leaf. The results of these methods were assimilated using an incremental classification algorithm.

Bashish et.al. [3] suggested a methodology for automatic detection and classification of plant leaf diseases with four main steps. In the first step they designed a color transformation structure for the RGB leaf image and then used device-independent color space transformation for the color transformation structure.In 
the second step, the images were segmented using the K- means clustering technique. In the third step, the texture features for the segmented infected objects were calculated. Finally, in the fourth step the features extracted, were passed through a pre-trained neural network.

Md. Zahangir Alom [2] recommended a new method based on Gaussian Mean (GM) for recognising paddy disease. The experimental results showed that the proposed method could automatically segment the diseased part of the paddy leaf robustly and accurately.

Patil et.al. [8] put forward Simple Threshold and Triangle Thresholding methods for segmenting leaf area and lesion region area respectively. Finally diseases were categorized by calculating the quotient of lesion area and leaf area. The accuracy of the experiment was found to be $98.6 \%$.

Tian et.al. [10] suggested a new method of Multi-Classifier System based on SVM for pattern recognition of wheat leaf diseases with high recognition accuracy. Diseased leaf samples with Powdery Mildew, Rust Puccinia Triticina, Leaf Blight, and Puccinia Striiformis were collected in the field and images were captured before a uniform black background. Three feature sets namely color feature set, shape feature set and texture feature set were created for classification analysis. The proposed strategy was based on a layered structure with two-levels: base- level was a module of three kinds of SVM-based classifiers trained by three feature sets and the meta-level was a module of SVM-based decision classifier trained by a meta-feature set generated through a new data combination mechanism.

$\mathrm{Li}$ et.al. [7] applied the method of automatic identification of three wheat diseases by analyzing the morphological characteristics extracted from their images. The target area was obtained by segmenting three kinds of wheat disease images of wheat powdery mildew, wheat sharp eyespot, and wheat stripe rust. Via extracting and optimizing the morphological data and using statistical analysis software to analyze the data with the principal component analysis and the discriminant analysis, five characteristic parameters such as Sphericity, Roundness, $\mathrm{Hu} 1, \mathrm{Hu} 2$, equivalent radius were selected as the identification factors. The recognizable rates of the samples among the three wheat diseases were $96.7 \%, 93.3 \%$, and $86.7 \%$ respectively.

\section{WHEAT CROP IMAGE ACQUISITION}

Images of the infected wheat plants are captured by closed circuit CCD cameras to cover approximately 3 sq. meter area that could acquire good quality images of wheat crop and processed for getting a gray colored image and then using image growing, image segmentation techniques to detect infected parts of the plants. Then the image is transferred to the analysis algorithm and report the quality. The methods evolved in this system are both image processing and soft computing technique applied on number of diseased wheat plant images.

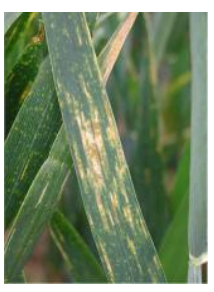

Fig. 1

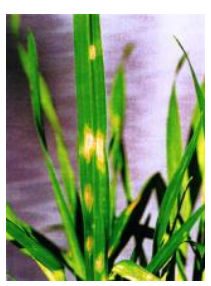

Fig. 2

\section{COLOR BASED SEGMENTATION}

Segmentation is a way to separate groups of objects. K-means clustering treats each object as having a location in space. It finds partitions such that objects within each cluster are as close to each other as possible, and as far from objects in other clusters as possible. K-means clustering requires that you specify the number of clusters to be partitioned and a distance metric to quantify how close two objects are to each other. In the presented work, color has been chosen as the criteria for clustering the wheat crop that will separate out the yellow part i.e. the yellow rust.

Following images shows the result after implementing the K-means algorithm on original wheat crop images.
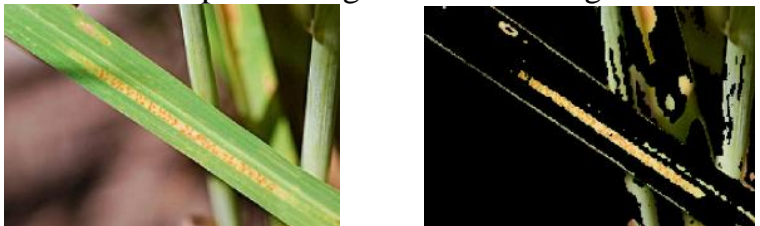

Fig. 3 (Original Wheat Crop) Fig. 4 (Segmented Yellow Rust) 


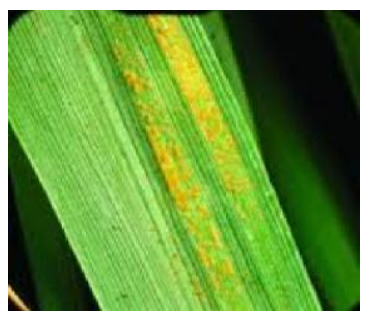

Fig. 5 (Original Wheat Crop)

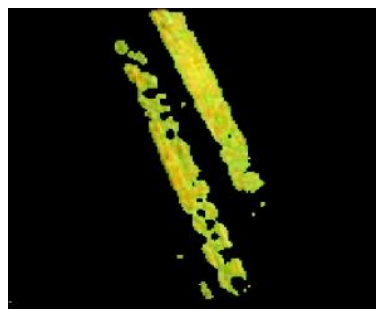

Fig. 6 (Segmented Yellow Rust)

\section{RESULTS}

The segmented image i.e. the yellow rust image is now exposed to measurement algorithm, where the yellow rust area is computed with respect to entire acquired image area. This gives the degree of yellow rust penetration in the wheat crop under scanner.

\section{CONCLUSION}

It can be observed from the resultant images, using color based image segmentation; it is possible to extract the yellow rust from the wheat crop images. Further, the segmented yellow rust images can be exposed to measurement algorithm where the actual penetration of the yellow rust may be estimated in the yield. This kind of image segmentation may be used for mapping the changes in land use land cover taken over temporal period in general but not in particular. The success of the segmentation and actual penetration of yellow rust mainly depend upon the positioning of the cameras installed in order to acquire the images from the field.

\section{REFERENCES}

[1] Abu-Naser S.S., K.A. Kashkash and M. Fayyad, 2010. "Developing an Expert System for Plant Disease Diagnosis", Journal of Artificial Intelligence, 3: 269- 276.

[2] Alom Md. Zahangir, and Hyo Jong Lee, 2010. "Gaussian Mean Based Paddy Disease Segmentation". The 3rd International Conference on Machine Vision (ICMV 2010).

[3] Bashish Dheeb Al Bashish, Malik Braik and Sulieman Bani-Ahmad, 2011. "Detection and Classification of Leaf Diseases using K-means-based Segmentation and Neural networksbased Classification". Information Technology Journal, 10: 267-275.

[4] Beghin T., J. S. Cope, P. Remagnino, \& S. Barman,2010, "Shape and texture based plant leaf classification", International Conference on Advanced Concepts for Intelligent Vision Systems (ACVIS), pp.345-353.

[5] Geng Ying, Li Miao, Yuan Yuan, Hu Zelin, 2009."A Study on the Method of Image Pre- processing for Recognition of Crop Diseases," International Conference on Advanced Computer Control, 2009,icacc, pp.202-206.

[6] H.Al-Hiary, S.Bani-Ahmad, M.Reyalat, M.Braik \&Z.AlRahamneh, 2011. Fast \& accurate detection classification of plant diseases, international journal of computer applications (0975-8887), volume 17no.1, pp-31-38.

[7] Li Jinghui, Lingwang Gao, Zuorui Shen, 2010.” Extraction and analysis of digital images feature of three kinds of wheat diseases". 3rd InternationalCongress on Image and Signal Processing (CISP2010).

[8] Patil Sanjay B., Dr. Shrikant K. Bodhe" Leaf Disease Severity Measurement Using Image Processing" International Journal of Engineering and Technology, Vol.3 (5), 2011,297-301.

[9] Sankarana Sindhuja, Ashish Mishra, Reza Ehsania."A review of advanced techniques for detecting plant diseases". Computers and Electronics in Agriculture 72 (2010) 1-13.v

[10] Yuan Tian, Chunjiang Zhao, Shenlian Lu, Xinyu Guo, 2009." Multiple classifier combination for recognition of wheat leaf diseases". Intelligent Automation and Soft Computing, Vol. 15, No. X, pp. 110, 2009.

[11] Mr. Viraj A. Gulhane, Dr. Ajay A. Gurjar," Detection of Diseases on Cotton Leaves and Its Possible Diagnosis", International Journal of Image Processing (IJIP), Volume (5): Issue (5): 2011.

\section{Author Profile}

Amina Bhaika received her M.Tech. degree in ECE from DIET, Kharar, Punjab in 2012. Her field of interest is in image processing based application systems. Presently the author is Asst. Prof. ECE in BGIET,Sangrur. 